\title{
THE GAMMA-RAY TELESCOPE GAMMA-1
}

- Akdimov,V.V., Nesterov,y.E., Kalinkin,L.F., Balibanov,V.M., Prilutsky,O.F., Rodin,V.G., Leikov, H.G., Bielaoussov,A.S., Dobrian,L.B. Poluektov,V.P., Gerassimov, I.A., Koslov V.D. Institute for Cosmic Research, Moscow (IKI)

- Voronov,S.A., Galper,A.M., Kirrilov-Ugriumov,V.G., Oserov,Y.V., Grigoriev,V.A., Iaurkine,Y.T., Popov,A.V. Moscow Institute of Physics (MIFI)

- Kurnosova,L.V., Fradkin,M.N. Physical Institute of the Academy of Sciences, Moscow (FIAN)

- Tsuikin,E.T. Leningrad Physical and Technical Institute (LFTI)

- Leray,J.P., Gros,M., Parlier,B., Soroka,F., Masse,P. S.A.P. / D.P.h, Centre d'Etudes Nucléaires, Saclay (France)

- Bazer-Bachi,A.R., Lavigne,J.M. Centre d'Etudes Spatiales des Rayonnements, Toulouse (France).

\section{ABSTRACT}

French and Soviet specialists have designed and built the gamma-ray telescope GAMMA-1 to detect cosmic gamma rays above $50 \mathrm{MeV}$. The sensitive area of the detector is $1400 \mathrm{~cm}^{2}$, energy resolution is $30 \%$ at $300 \mathrm{MeV}$, and angular resolution $1.2^{\circ}$ at $300 \mathrm{MeV}$ (and less than $20^{\prime}$ arc when a coded aperture mask is used). Results on calibration of the qualification model and Monte-Carlo calculations are presented.

1. Introduction. SAS.II, then the long-lived COS-B, have shown the interest of high-energy gamma ray astronomy. Numerous point sources have been found, but identification with physical objects has been possible for only a few of them. The first objective of the GAMMA-1 experiment was to increase the sensitive area, and lower the angular resolution of a gamma ray detector. A wide gap spark chamber, tested at DESY in $1976(1,2)$, showed that it was possible to obtain better than $2^{\circ}$ angular resolution with this technique.

However, in order to still increase this resolution, a model of coded aperture mask was developped (3). In a spark chamber, image deconvolution is performed not only by position detectors, but. also by taking into account the rough $\left(\mathrm{C}^{\circ}\right)$ arrival direction information. The addition of this mask, and the complexity of the experiment itse if, resulted in some delay over the initial launch date.

2. The experiment. The Gamma-1 telescope is illustrated on Fig.1. The main detector is a $50 \times 50 \mathrm{~cm}, 12-1$ ayered wide gap spark chamber. It is shielded with lateral and upper anticoincidence, inside and outside the satellite skin. A time of flight $(S V+S N)$ can veto upgoing particles, 


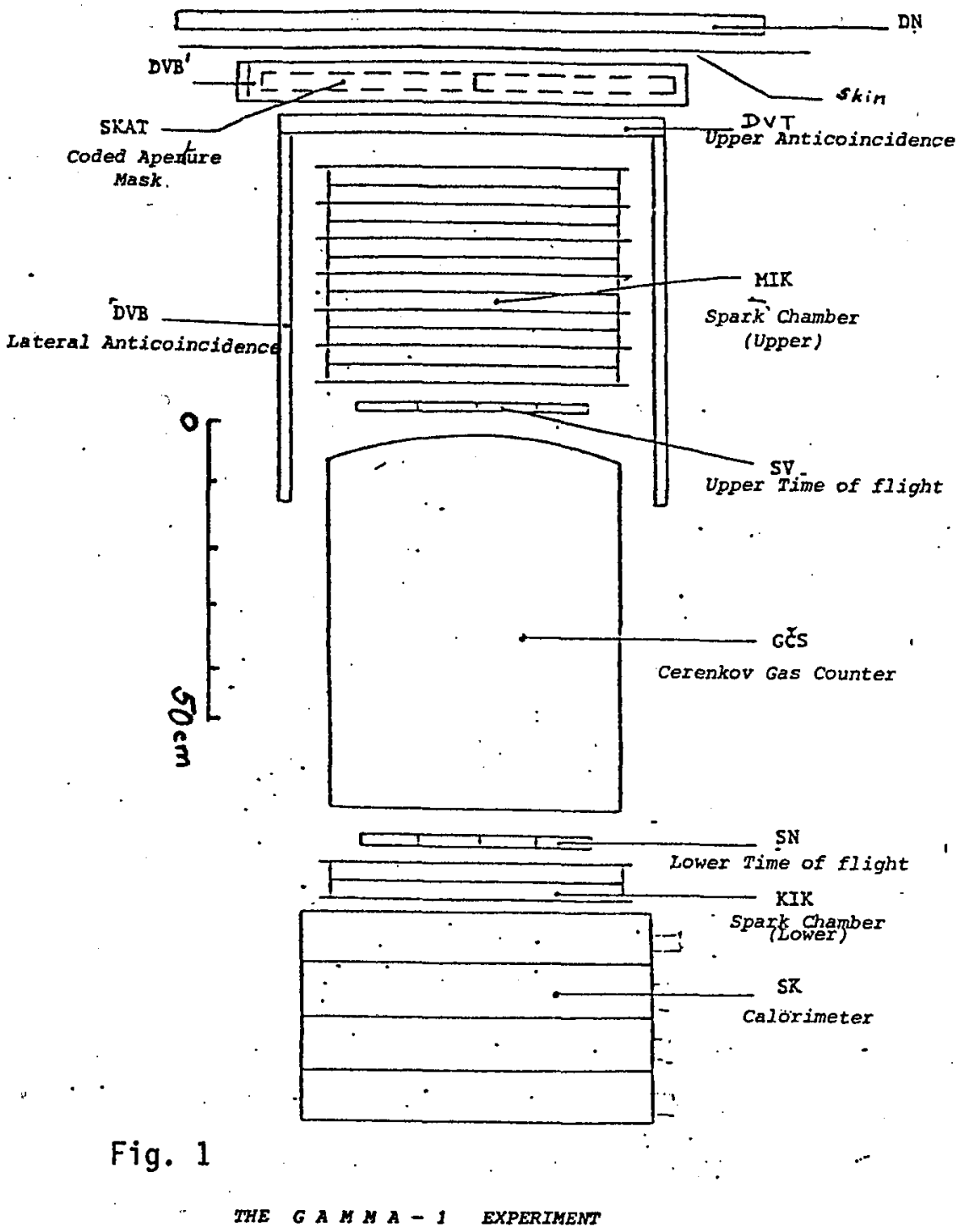

in reduddance with a Cerenkov gas counter. A two layered spark chamber below the SN counter is helpful when the very high energy electronpositron pair cannot be separated in the upper chamber.

To measure the energy, scattering is used from 50 to $200 \mathrm{MeV}$, and a four-layered-calorimeter is used above $100 \mathrm{MeV}$.

The spark images are viewed through an optical system by two orthogonal vidicon cameras and digitised before storage on a recorder. The $50 \mathrm{~cm}$ width of the chamber is divided in4096 points (localisation laccuracy $0.125 \mathrm{~mm}$ ). For each spark, there are three lines of sweep, separated by $17 \mathrm{~mm}$; the width of each digitised spark is also recorded. The dead time of the high voltage (24 KV) generator is less than $0.5 \mathrm{sec}$. Data from the vidicons, and from all counters, along with housekeeping informations, are dumped to the telemetry station twice a day. 
The coded mask is made of two orthogonal one-dimensionnal arrays of $1 \mathrm{~cm}$-thick tungsten bar,each one covering half of the field of view, which can slide on or off the field of view of the experiment. The experiment can be put in many different modes by telecommands (for instance, recording of proton tracks to check linearity of the imaging system.) while the satellite is above the station.

3. Calibration. The qualification model has been extensively calibrated

in the tagged gamma ray beam 6500

THETA $=0$

of the accelerator "Pakhra", near Moscow. The set-up of the beam has been described elsewhere

Fig.2

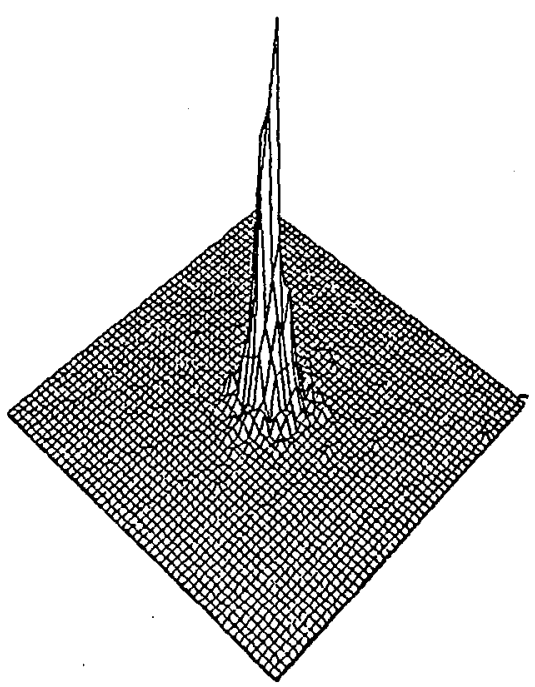

(4.), but Fig. 2 shows arrival directions of 1000 gamma rays on top of the chamber, on axis (bin size $1 \mathrm{~cm}$ ). FWHM is about $4 \mathrm{~cm}$. The energy resolution, from 30 to $700 \mathrm{MeV}$, is about $30 \mathrm{MeV}$, and the openning of the beam 2.5 mrad.

In parallel, Monte-Carlo calculations were performed, to check the actual response up to $700 \mathrm{MeV}$, and to be used as calibration above.

Figures 3 and 4 show the efficiency and angular resolution of the detector, without the coded mask. Energy resolution ( as measured by the calorimeter), decreases from $95 \%$ at $50 \mathrm{MeV}$ to $30 \%$ at $500 \mathrm{MeV}$.

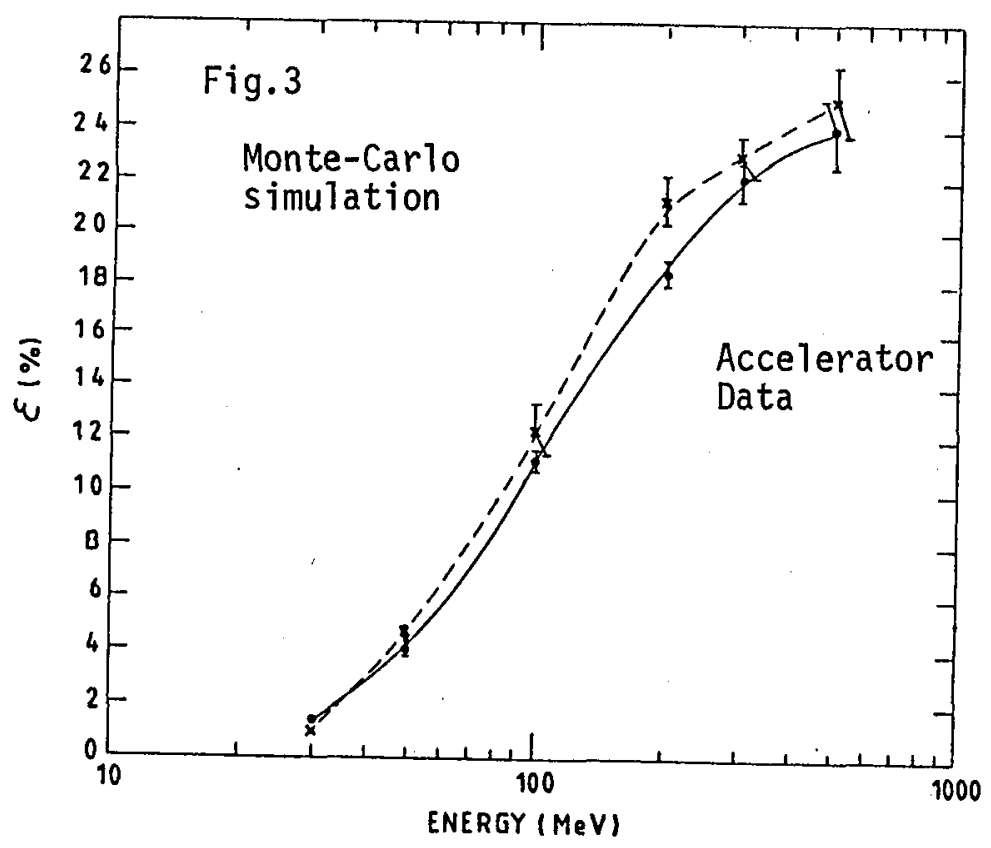

Calibrations have also been performed using the mask. An exemple of correlation function at $300 \mathrm{M} \mathrm{eV}$ is given in Fig. 5 . Bin size is $5^{\prime}$ arc. The actual position of the beam was at $-15^{\prime}$. Reconstructed direction is $(-10 \pm 5)^{\prime}$ arc, with a FWHM of $28^{\prime}$. $T$ Such a figure may seem high, but when one takes into account the angular width of the beam itself, the Fh:HM is less than $15^{\prime}$ of arc. 


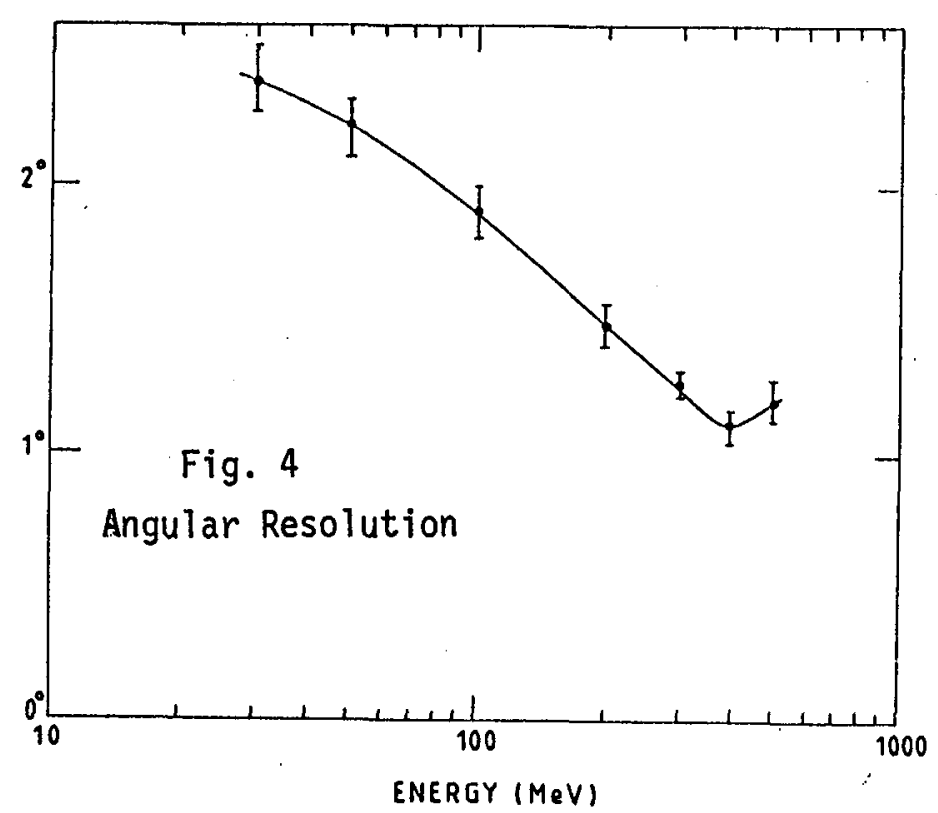

4: Conclusions. The calibrations performed on the qualification mode1 of the Gamma-1 experiment, as well as calculations, have shown its potential in reconstructing gammarays directions. The use of a coded mask can give images with $15^{\prime}$ arc resolution. Absclute celestial coordinates are obtained with the helf of a stellar sensor with $5^{\prime}$ arc accuracy. Without the mask, upper flux limits of $10^{-7}$

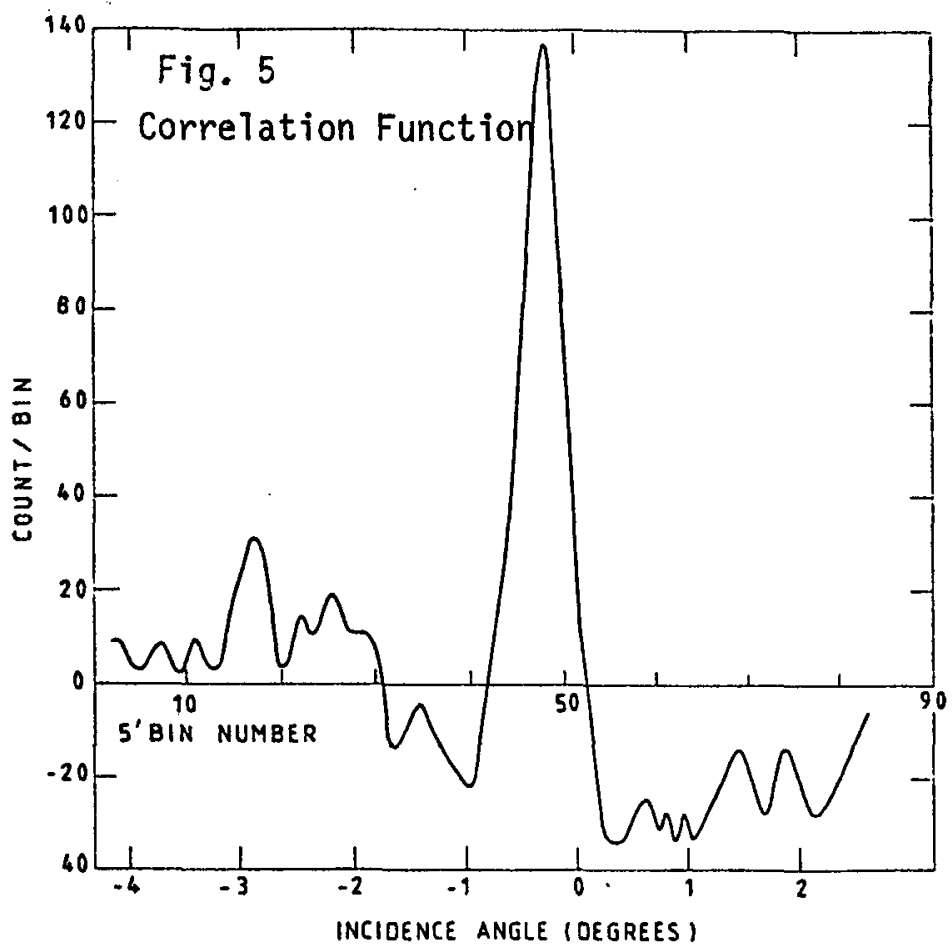
should be detected with in two weeks of exposure, using a sensitive area of $1400 \mathrm{CM}^{2}$, and gamma rays within $10^{\circ}$ of the axis. Calibration of the flight model will be performed at the end of 1985 , on a reduced program. Then, it will be integrated in a Salyut-type, 3-axes stabilised, satellite, to be launched in late 86 on a low-altitude orbit.

References. 1- Akimov, V.V. et al\% Nuciear Inst. \& "iet., 147, 329 (1977) 2.- Bazer-Bachi, A.R., et al, Proc 12th Es7ab Symposium, Frascati(1977) 3.- Prilutsky, O.F., Proc XXI I COSPAR Meeting, Bangalore (1979)

4.- Zverev,V.G., et al, Atomizdat 1980, p45 (Moscow) 